\title{
Polyacrylamide gel substrates that simulate the mechanical stiffness of normal and malignant neuronal tissues increase protoporphyin IX synthesis in glioma cells
}

Carolyn J. Niu

Carl Fisher

Kira Scheffler

Rachel Wan

Hoda Maleki

Haijiao Liu

Yu Sun

Craig A. Simmons

Reginald Birngruber

Lothar Lilge

\section{SPIE.}




\title{
Polyacrylamide gel substrates that simulate the mechanical stiffness of normal and malignant neuronal tissues increase protoporphyin IX synthesis in glioma cells
}

\author{
Carolyn J. Niu, ${ }^{a}$ Carl Fisher, ${ }^{b}$ Kira Scheffler, ${ }^{a}$ Rachel Wan, ${ }^{a}$ Hoda Maleki, ${ }^{c}$ Haijiao Liu, ${ }^{c}$ Yu Sun, ${ }^{c}$ \\ Craig A. Simmons, ${ }^{c}$ Reginald Birngruber, ${ }^{d}$ and Lothar Lilge ${ }^{a, b, *}$ \\ aPrincess Margaret Cancer Centre, 101 College Street, Toronto, Ontario M5G1L7, Canada \\ bUniversity of Toronto, Department of Medical Biophysics, 101 College Street, Toronto, Ontario M5G1L7, Canada \\ 'University of Toronto, Department of Mechanical and Industrial Engineering, 5 King's College Road, Toronto, Ontario M5S3G8, Canada \\ dUniversität zu Lübeck, Institut für Biomedizinische Optik, Peter-Monnik-Weg 4, 23562 Lübeck, Germany
}

\begin{abstract}
Protoporphyrin IX (PPIX) produced following the administration of exogenous 5d-aminolevulinic acid is clinically approved for photodynamic therapy and fluorescence-guided resection in various jurisdictions around the world. For both applications, quantification of PPIX forms the basis for accurate therapeutic dose calculation and identification of malignant tissues for resection. While it is well established that the PPIX synthesis and accumulation rates are subject to the cell's biochemical microenvironment, the effect of the physical microenvironment, such as matrix stiffness, has received little attention to date. Here we studied the proliferation rate and PPIX synthesis and accumulation in two glioma cell lines U373 and U118 cultured under five different substrate conditions, including the conventional tissue culture plastic and polyacrylamide gels that simulated tissue stiffness of normal brain $(1 \mathrm{kPa})$ and glioblastoma tumors (12 $\mathrm{kPa})$. We found that the proliferation rate increased with substrate stiffness for both cell lines, but not in a linear fashion. PPIX concentration was significantly higher in cells cultured on tissue-simulating gels than on the much stiffer tissue culture plastic for both cell lines. These findings, albeit preliminary, suggest that the physical microenvironment might be an important determinant of tumor aggressiveness and PPIX synthesis in glioma cells. @ 2015 Society of Photo-Optical Instrumentation Engineers (SPIE) [DOI: 10.1117/1.JBO.20.9.098002]
\end{abstract}

Keywords: glioblastoma; photodynamic therapy; substrate stiffness.

Paper 150399R received Jun. 11, 2015; accepted for publication Aug. 28, 2015; published online Sep. 25, 2015.

\section{Introduction}

Photodynamic therapy (PDT) mediated by a variety of photosensitizers is under intense investigation as standalone or adjuvant therapy for the management of malignant glioblastoma. ${ }^{1,2}$ A considerable number of clinical trials have been conducted with some very promising results for hematoporphyrin derivative ${ }^{3}$ aminolevulinic acid (ALA)-protoporphyrin IX (PPIX), ${ }^{4,5}$ and talaporfin ${ }^{6}$ mediated PDT. In particular, in ALA-PPIX mediated PDT, ALA is administered as the pro-drug, which is converted into the active photosensitizer PPIX along the hemebiosynthesis pathway when the particular glioma has the ability to do so. ${ }^{7}$ ALA-PPIX mediated PDT has been shown to promote desirable clinical outcomes by reducing tumor size or controlling tumor growth, thus, providing a survival advantage. ${ }^{4}$ On a macroscopic scale, it was clearly demonstrated that tumor response to ALA-PPIX mediated PDT is closely related to average PPIX concentration at the site of PDT treatment, in concordance with established PDT dose models. ${ }^{8}{ }^{8}$ PPIX concentration can be assessed locally via its fluorescent properties by point spectroscopic methods ${ }^{10}$ or imaging modalities. ${ }^{11}$ These fluorescence-based assessment techniques are also the basis for PPIX mediated fluorescence guided resection (FGR) of malignant glioma and other brain tumors, which has been proven to be a highly effective adjuvant therapy independent of PDT. ${ }^{12}$ One caveat of FGR though is the requirement that the fluorescent quantum yield of PPIX and, to a lesser impact, its spectral emission property are independent of physiological changes in the tissue microenvironment, such as $\mathrm{pH}$ and partial oxygen pressure, assumptions shown to not always be true..$^{13,14}$

One challenge toward quantitative ALA-PPIX mediated PDT and FGR is the large variability in the amount of PPIX synthesized between different cell lines ${ }^{15}$ and between patients. ${ }^{16}$ For PPIX based FGR, other factors such as local tissue optical properties and alteration to the PPIX fluorescence quantum yield due to microenvironment change further complicate quantification of the perceived PPIX fluorescence in terms of absolute in vivo PPIX concentration. ${ }^{17}$ Thus, clinical FGR to date still largely relies on qualitative descriptors such as strong or vague fluorescence. ${ }^{12}$ Nevertheless, numerous groups are actively developing quantitative fluorescence measurement in clinical trials. ${ }^{11,18,19}$ For both FGR and PDT, the preferential accumulation and retention of PPIX in the tumor compared to the surrounding tissue are a prerequisite to their successful application. ${ }^{20}$ Other factors such as subcellular distribution of PPIX are of interest predominantly to PDT. The importance 
of the mitochondria in the cytotoxicity of ALA-induced-PPIX mediated PDT was adequately reviewed by Amo et al. ${ }^{21}$ In addition, Kobuchi et al. observed that ATP-binding cassette transporter G2 (ABCG2) localized in the mitochondria exports PPIX from the mitochondria into the cytosol. ${ }^{22}$ Subsequently, Yamamoto et al. found that the cell killing efficacy of ALAPPIX could be improved by adding ABCG2 inhibitors, further supporting the premise that mitochondrial PPIX is more cytotoxic than cytosol PPIX. ${ }^{23}$

One additional limitation, particularly for ALA-PPIX mediated PDT, is the maximum permissible ALA dose administered to patients. In current clinical protocols, ALA is administered orally at a dose of 20 to $40 \mathrm{mgkg},{ }^{2,4}$ resulting in an insufficient PPIX concentration in the tumor tissue in a notable fraction of the patient population, potentially leading to treatment failure. ${ }^{16}$ The variability in the attainable PPIX concentration makes it exceedingly difficult to identify patients suitable for ALAPPIX mediated PDT or FGR based solely on the tumor's genetic traits. It is of great clinical interest to determine factors, intraand extracellular, that affect the cell's ability to synthesize PPIX, as well as the fashion in which these factors act. This knowledge can also lead to the discovery of novel methods and means to manipulate PPIX production in a favorable manner to enhance PDT treatment selectivity and, hence, improve outcome while reducing morbidity.

It has long been known that cells interact with their surroundings in an intimate and tightly regulated manner through constant remodeling of and receiving signals from the extracellular matrix $(\mathrm{ECM}){ }^{20}$ In particular, the mechanical properties of the ECM have attracted considerable attention in recent years. ${ }^{24}$ It has been shown that substrate stiffness alone is sufficient to determine the differentiation fate of stem cells in vitro. ${ }^{25}$ Studies have also shown that tissue stiffness increases prior to any tumor mass becoming visible. ${ }^{26}$

Although the crucial role of a cell's mechanical environment has been established, the vast majority of published in vitro studies on glioma were conducted on traditional tissue culture plastic (TCP) of which the Young's modulus is in the range of $10 \mathrm{MPa}$, many orders of magnitude higher than that of actual brain tissue, which has been shown to be in the kPa range. ${ }^{27,28}$ Therefore, in the context of ALA-PPIX mediated PDT and FGR to treat glioma, the aim of this study is to evaluate the effect of substrate stiffness on the proliferation of and PPIX synthesis in glioma cells. To address this aim, in vitro experiments were carried out on substrates with stiffness similar to that of normal brain tissue and glioma tumors. The same experiments were also conducted using cells grown on standard TCP to serve as the baseline for comparison.

This study investigated the effect of substrate stiffness on the proliferation of glioma cells and PPIX synthesis in two human glioma cell lines, U118 and U373. A previous study conducted by the investigators to study PPIX synthesis in 10 cell types showed that U373 was a comparably high PPIX producer, whereas U118 synthesized little PPIX. ${ }^{15}$ Therefore, these two cell lines were chosen for this study to represent the range of glioma cell lines in terms of PPIX synthesis. Since PPIX localized within the mitochondria is expected to generate stronger cytotoxic effects during PDT than when diffusely distributed in the cytoplasm, this study focused on the PPIX concentration inside the mitochondria, which was determined semiquantitatively via fluorescence while costaining mitochondria during live cell imaging.

\section{Materials and Methods}

\subsection{Substrate Preparation}

Two human glioma cell lines U373 and U118 were examined under five different mechanical conditions in this study. The baseline condition was the conventional TCP, providing the stiffest substrate surface.

Softer substrates, made of polyacrylamide, aimed to simulate the Young's moduli of normal brain and glioma. The polyacrylamide substrates were produced following a protocol described by Fischer et al. ${ }^{29}$ In short, $12 \mathrm{~mm}$ diameter glass coverslips (Electro Microscopy Sciences, Hatfield, Pennsylvania), each supporting one substrate, were first washed with Piranha acid. The coverslip surfaces were activated with $0.5 \%$ 3-aminopropyltrimethoxy silane (Sigma-Aldrich, Mississauga, Ontario, Canada) and $0.5 \%$ glutaraldehyde (Sigma-Aldrich, Mississauga). Polyacrylamide gels of two different stiffnesses were formed on the activated surface. Normal brain representing gels were produced by polymerizing 3\% acrylamides (Bio-Rad Laboratories, Mississauga, Ontario) and $0.1 \%$ bis-acrylamide (Bio-Rad Laboratories) dissolved in double distilled water. The stiffer glioma representing gels were produced by $7.5 \%$ acrylamide and $0.15 \%$ bis-acrylamide. If gels were not used immediately after fabrication, they were stored at $4^{\circ} \mathrm{C}$ under $100 \%$ humidity for up to one week. Gels produced by this protocol were 25 to $50 \mu \mathrm{m}$ thick. It has been suggested that cells grown on top of the gel would be able to sense the rigid coverslip underneath if the gel is $<20 \mu$ thick. $^{29}$

For cells to grow, the gels were coated with collagen which acted as the scaffold that cells could anchor onto. Collagen was chosen as the coating ECM protein because it is commonplace in most connective tissues and its interactions with cells are well studied and well understood. ${ }^{20}$

To overlay collagen on top of the gel, $200 \mu \mathrm{L}$ of hetero bifunctional-cross-linker Sulfo-SANPAH (Sigma Aldrich, Mississauga) was added on top of the gels and activated by UV light (UVO Cleaner, JetLight Company Inc., Irvine, California) for $30 \mathrm{~s}$ at an irradiance of 28 to $32 \mathrm{mWcm}^{-2}$. After that, $75 \mu \mathrm{L}$ of $100 \mathrm{mgmL}^{-1}$ rat tail collagen I (BD Biosciences, San Jose, California) in phosphate buffered saline solution was dropped onto each gel. The mixture was left for a minimum of $4 \mathrm{~h}$ at room temperature to link the collagen to the gel via the cross-linker Sulfo-SANPAH.

For both cell lines, 70,000 cells in $35 \mu \mathrm{L}$ suspension were seeded on each collagen coated substrate. For half of the seeded substrates, no further manipulation of the mechanical environment was performed. For the other half, another layer of collagen $\left(2 \mathrm{mgml}^{-1}\right.$ rat tail collagen I solution) was placed on top of the cells $24 \mathrm{~h}$ after seeding, so that these cells were sandwiched between two collagen layers acting as extracellular scaffolds to more closely model the three-dimensional (3-D) in vivo environment. This structure is referred to as the 3-D condition, whereas the cells without the coverage of the top collagen layer are referred to as growing in the two-dimensional (2-D) condition.

It should be mentioned here that since all tissue-mimicking gels were coated with a layer of collagen before cells were seeded, the ideal baseline material should also be covered by a layer of collagen. The authors had attempted to coat collagen directly onto glass coverslips, but were not able to keep the collagen coating from falling off the coverslip for more than two days. Because of this procedural limitation, TCP, which did not require any additional coating to support cell growth, was used 
as the rigid baseline substrate. The absence of a collagen layer on TCP is acknowledged by the authors and is a methodological issue to be addressed in the future.

Before in vitro investigation was carried out, the Young's moduli of the brain and tumor representing polyacrylamide gels (without the collagen overlay) were measured daily over three consecutive days using atomic force microscopy (AFM) (BioScope Catalyst, Bruker, Santa Barbara, California) to observe any potential change in gel stiffness over time. The stiffness measurement protocol is described in detail by Liu et al. in the supplementary materials to their publication. ${ }^{30}$ On each day, the Young's modulus of each gel was measured at four random locations across the gel surface, with three repeats at each location.

\subsection{Cell Proliferation under Different Mechanical Conditions}

Cells cultured on TCP, 2-D brain, and 2-D tumor simulating gels were imaged and counted daily for four days after seeding to determine if and how substrate stiffness affects cell density change rate. CellTracker Red CMTPX (Life Technologies, Burlington, Ontario, Canada), a fluorescent dye that is retained inside cells over multiple generations, was used to track cell density over time. Cells were stained on day one by incubating them for $30 \mathrm{~min}$ in $1: 1000$ CellTracker Red solutions. To count, cells were imaged using a $10 \times$ objective on an inverted fluorescence microscope (AxioObserver, $10 \times / 0.30$ EC Plan-Neofluar lens, Carl Zeiss, Goettingen, Germany) using a $550 \mathrm{~nm}$ excitation filter and a $610 \mathrm{~nm}$ band pass emission filter. Nine fields-ofview (FOVs) were imaged per cell line per substrate per day to observe the change in cell density as a function of substrate over time. Each FOV captured an area of $1.34 \mathrm{~mm}^{2}$, and the cells within each FOV were counted manually. For each cell line and each substrate, the average number of cells across all nine FOVs acquired on the same day was calculated and normalized to the initial cell count on day one.

\subsection{Live Cell Imaging to Determine Protoporphyrin IX Production in the Mitochondria}

PPIX production in cells grown in the five biomechanical conditions (TCP, 2-D brain simulating gel, 2-D glioma simulating gel, 3-D brain simulating gel, and 3-D glioma simulating gel) was determined using quantitative fluorescence microscopy as described by Fisher et al., where PPIX fluorescence intensity is considered a surrogate for PPIX concentration. ${ }^{15}$ Briefly, cells were cultured for $48 \mathrm{~h}$ on the different substrates to allow any stiffness induced cellular change to take place. Cells were then incubated at an ALA concentration of $1 \mathrm{mM}$ for $4 \mathrm{~h}$, the same incubation period in clinical applications, during which PPIX was synthesized and accumulated. The same incubation media also had $500 \mathrm{nM}$ MitoTracker Red (MTR), a fluorescent dye that labels the mitochondria.

Imaging was performed using an inverted fluorescence microscope that enables live cell imaging (Zeiss AxioObserver, Carl Zeiss MicroImaging $\mathrm{GmbH}$, Gottingen, Germany). The objective was a $40 \times$ oil immersion lens with $\mathrm{NA}=1.3$ (EC Plan-NeoFluar Carl Zeiss MicroImaging $\mathrm{GmbH}$ ), yielding an FOV dimension of $224 \times 168 \mu \mathrm{m}^{2}$. The camera has a $1388 \times$ 1040 pixel array, equivalent to 6.2 pixels per $\mu \mathrm{m}$. Considering that mitochondrial width ranges from 0.5 to $1 \mu \mathrm{m},{ }^{31}$ each mitochondrion would be imaged by $\sim 3$ to 6 pixels along its width.
MTR was imaged using a $590 \mathrm{~nm}$ excitation light-emitting diode and the emission was collected via a 610 to $650 \mathrm{~nm}$ band pass filter. PPIX fluorescence was imaged using $405 \mathrm{~nm}$ excitation filter and a $594 \mathrm{~nm}$ long pass emission filter. In order to minimize photobleaching of PPIX, searching for FOVs and focusing were done at the $590 \mathrm{~nm}$ MTR excitation light, of which PPIX absorbs very little. Standard non-photobleaching fluorescence beads were imaged in both the MTR and PPIX channels on each imaging day to correct for the day-to-day fluctuation in the microscope's light throughput. Additional cellfree background images of the substrates were acquired to account for the nonuniform illumination across the FOV. Eight FOVs were captured per cell line per substrate.

A MATLAB ${ }^{\circledR}$ (MathWorks, Woburn, Massachusetts) program was written to analyze the fluorescence images. Since PPIX concentration within the mitochondria is of interest, the mitochondria were delineated on the MTR images. First, the background acquired on the same imaging day was removed from each MTR image to account for nonuniform illumination. After background removal, image intensity was normalized by the average fluorescence intensity of the standard beads captured on the same imaging day. The process to standardize and correct each MTR image is summarized by Eq. (1):

$I_{\mathrm{MTR} \text { corrected }}=\frac{I_{\mathrm{MTR} \text { raw }}-\text { Background }_{\mathrm{MTR}}}{\text { Standard }_{\mathrm{MTR}}}$,

where $I_{\mathrm{MTR} \text { corrected }}$ refers to the corrected MTR image; $I_{\mathrm{MTR}}$ raw refers to the raw MTR image; Background ${ }_{\text {MTR }}$ refers to the cellfree background image in the MTR channel; and Standard MTR $_{\text {}}$ refers to the average fluorescence intensity of the standard beads.

A mask, which has the same size as the MTR image, to delineate the mitochondria was then generated by selecting all pixels above a certain intensity threshold in each MTR image. The threshold for each image was calculated using Otsu's method. ${ }^{32}$ The process to generate the mask is summarized by Eq. (2):

Mask $_{\text {Mito }}=\left\{I_{\text {MTR corrected }} \geq\right.$ Threshold $\left._{\text {MTR Otsu }}\right\}$,

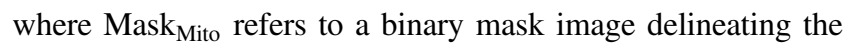
mitochondria in the corrected MTR fluorescence images. Threshold $_{\text {MTR Otsu }}$ is the intensity threshold calculated using the Otsu's method. If a pixel in $I_{\text {MTR corrected was greater than or }}$ equal to Threshold $\mathrm{MTR}_{\mathrm{MTsu}}$, the corresponding pixel in Mask $\mathrm{Mito}_{\text {}}$ would be 1 , otherwise 0 .

The PPIX fluorescence images were also corrected with the background image and standard fluorescence in the PPIX channel in the same manner as the MTR images. The corrected PPIX images were then multiplied pixel-by-pixel with the corresponding mitochondria masks to obtain images of the PPIX fluorescence in the mitochondria only.

It was observed that cells grown on conventional TCP developed a flat, spread-out shape with an extended cytoskeleton and the majority of mitochondria were discernible individually throughout the cytoplasm with little overlap. In this situation, PPIX fluorescence intensity at each pixel could be employed as a surrogate for the local PPIX concentration. In the present study, cells cultured on soft gel substrates developed a spherical shape, possibly because they had grown into the soft gel instead of over the surface of the gel. For these spherical-looking cells, the fluorescence intensity at one pixel in a PPIX image could not be attributed to particular mitochondria, but originated from 
multiple overlapping mitochondria. For this reason, individual pixel intensity could not be considered an accurate representation of the local PPIX concentration. However, the total PPIX fluorescence counts still remained a valid representation of the total amount of PPIX in all mitochondria. Therefore, for each cell line and each substrate condition, the total PPIX fluorescence intensities were summed in all mitochondrial PPIX images. Additionally, the total number of cells captured in each image was also counted. The total PPIX fluorescence intensity was divided by the total number of cells to obtain the average mitochondrial PPIX per cell for that particular cell line and substrate condition.

\section{Results and Discussions}

\subsection{Substrate Stiffness}

The stiffness of both the brain and glioma simulating gels did not change significantly over a period of three days. The Young's moduli measured on the third day after fabrication of both gels are shown in Fig. 1. The average Young's modulus of the softer, normal brain simulating, gel is close to $1.0 \mathrm{kPa}$, with measurements at different locations across the gel surface ranging from 0.4 to $1.4 \mathrm{kPa}$. The average measured Young's modulus of the stiffer glioma representing gel was $12.3 \mathrm{kPa}$, with a range from 10 to $16 \mathrm{kPa}$.

The variability of stiffness measurements across a gel surface could be caused by micrometer-sized air bubbles formed during gel fabrication prior to curing completion. Since AFM achieves nano-scale spatial resolution, proximity of the highly sensitive AFM probe tip to such a bubble affects the determined Young's modulus. Additionally, the measurements were not conducted in a dust-free environment and tiny air-borne particles landing on the gels could also affect results. However, this is expected to be only a minor contribution to the PPIX measurement variability as the cells integrate the Young's modulus over their contact area.

\subsection{Cell Density Changes Under Different Mechanical Conditions}

The average number of cells per FOV counted over four days after seeding on the three growth substrates (TCP, 2-D brain

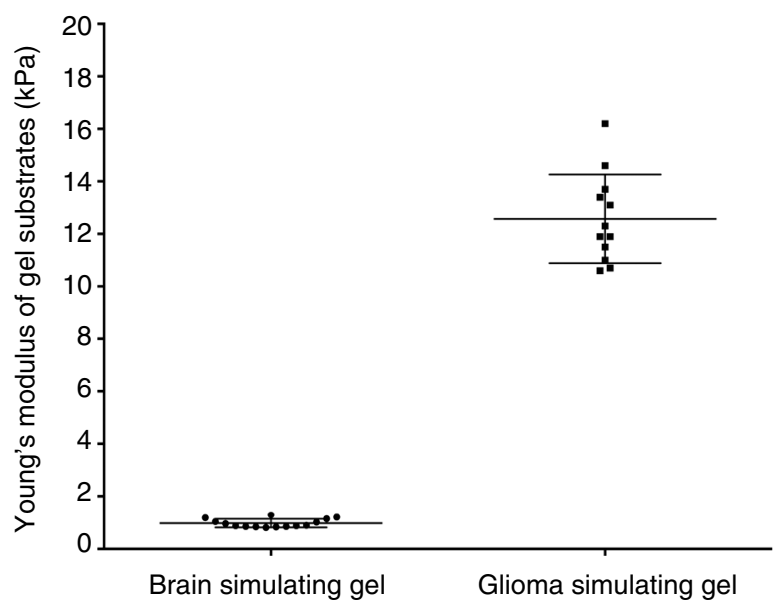

Fig. 1 Young's modulus of the normal brain simulating and glioma simulating polyacrylamide gels measured with atomic force microscopy three days after gel fabrication. simulating gel, and 2-D glioma simulating gel) is shown in Figs. 2(a) and 2(b) for the two cell lines, U118 and U373, respectively. The cell counts are normalized to the count on day one when at least 100 cells populated each FOV. The error bars are standard deviations in normalized cell count across all FOVs for each cell line and substrate condition. For easiness of viewing, for both cell lines only the top half of error bars is shown for glioma simulating substrate and the bottom half of error bars for brain simulating substrate. Since cells were counted over only four days, it was assumed that cell density increased in a linear fashion during this period. The normalized rates of apparent growth per day of U118 on TCP, glioma simulating, and brain simulating gels were $0.64,0.59$, and 0.52 , respectively. However, for U118, the difference in apparent growth rate on the various substrates was not statistically significant. U373 grew significantly faster on TCP than on either gel $(p<0.001)$, with the normalized growth rate on TCP, glioma simulating, and brain simulating gels being $1.09,0.57$, and 0.56 , respectively. The two cell lines seemed to grow the slowest on the softest brain simulating substrate and fastest on the stiffest TC plastic. For U118, its growth rate on the glioma simulating
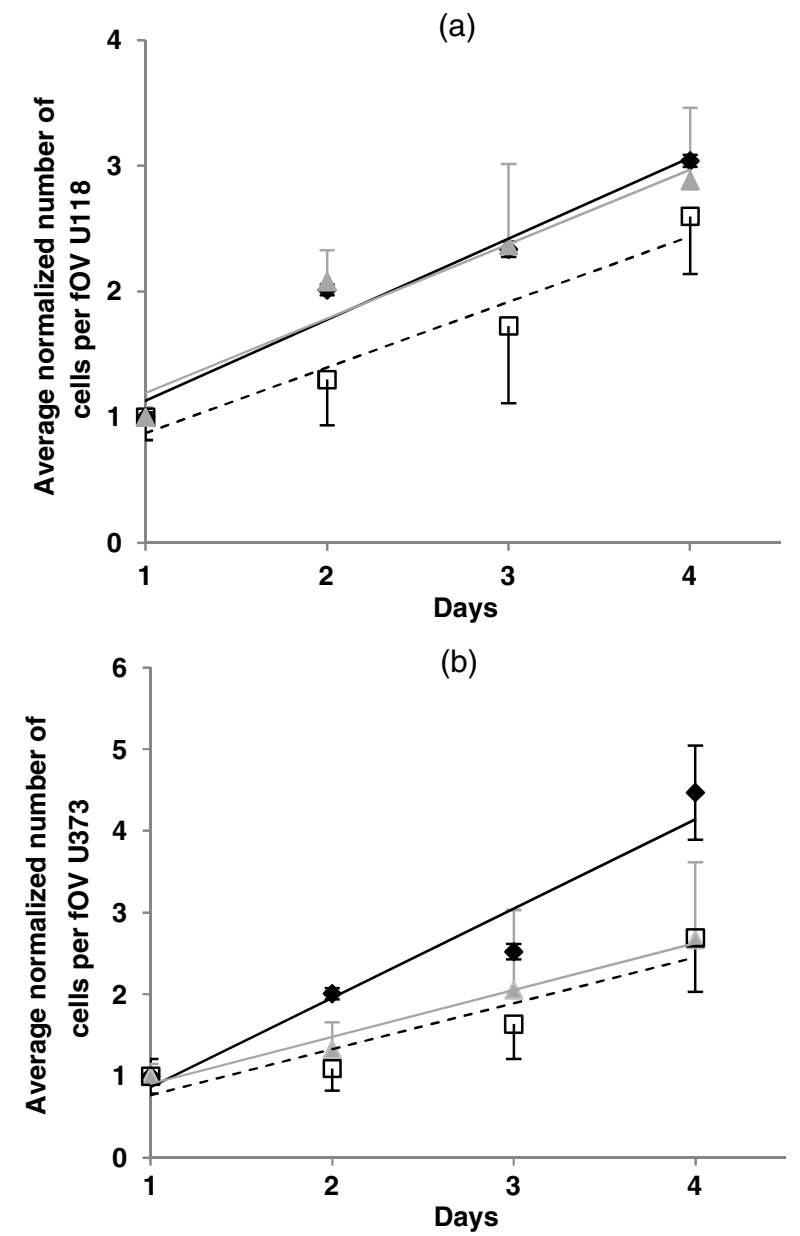

Fig. 2 Average number of cells per field of view, normalized to the average number of cells counted on the first day, on three substrates: tissue culture plastic (TCP) (solid black rhombus), two-dimensional (2-D) brain simulating (open square), and 2-D glioma simulating (gray triangles) gels, counted daily over four days postseeding. For clarity of viewing, only the top half of error bars is displayed for glioma simulating gel, and the bottom half of error bars is displayed for brain simulating gel: (a) U118 and (b) U373. 
gel was slightly closer to that on TCP than to that on the brain simulating gel. In contrast, the apparent growth rate of U373 on glioma simulating gel was almost the same as that on brain simulating gel.

Previous studies have reported that glioma cells proliferate at distinct rates on substrates of different Young's moduli. One proposed explanation is that ECM stiffness provides mechanical cues that modify cytoskeletal activities and coordination, which in turn are extensively involved in mitosis. ${ }^{27,33}$ Another postulation is that substrate stiffness can change proliferation via the epidermal growth factor receptor (EGFR) signaling pathway, given that EGFR is frequently upregulated in glioma. ${ }^{34}$ Since EGFR expression level is indicative of glioma malignancy, ${ }^{35}$ it is reasonable to speculate that more aggressive glioma could be more sensitive to changes in its biomechanical environment. However, the exact manner of this response is yet to be elucidated. It should be noted that both U373 and U118 are EGFR positive. ${ }^{15}$ This study observed the effect of substrate stiffness on cell proliferation, but also suggested that the degree to which proliferation is influenced does not seem to be linear with substrate stiffness. It seems possible that there exists a required minimum stiffness beyond which the substrate starts to stimulate glioma cell proliferation. For U118, that threshold appears to be closer to the Young's modulus of normal brain $(\sim 1 \mathrm{kPa})$ but higher $(>10 \mathrm{kPa})$ for U373. This again highlights the difference between cell lines of the same cancer, and subsequently the possible need to assess the mechanical properties of the bulk tumor, invasive front, and the surrounding normal tissue for malignancy in the brain and other organs as well. Noninvasive elastography techniques that employ existing medical imaging modalities, including ultrasound, ${ }^{36}$ magnetic resonance imaging, ${ }^{37}$ and optical coherence tomography, ${ }^{38}$ have already been under active development to achieve this goal.

Another observation is that cells cultured on TCP, the stiffest substrate, grew more uniformly over the substrate surface than the cells cultured on the softer gel substrates, as demonstrated by the larger standard deviation in the number of cells for the gel substrates. This could be attributed to spatial variations in the gel properties or their effect on cell proliferation and migration.

\subsection{PPIX Production in the Mitochondria}

The average mitochondrial fluorescence counts per cell, which represents the average relative PPIX concentration in the mitochondria per cell, is shown in Figs. 3(a) and 3(b) as a function of substrate condition for the two cell lines U118 and U373, respectively. A Student's $t$ test was performed between the PPIX fluorescence data acquired in any two substrate conditions to determine the statistical significance in PPIX fluorescence change as a result of substrate condition. Overall, both cell lines produced significantly more PPIX when grown on either gel substrates in the 2-D or 3-D approach, compared to the conventional TCP $(p<0.05)$. However, the two cell lines responded differently in terms of PPIX production to different gel constructed substrates. In particular, U118 produced and accumulated significantly more PPIX in the mitochondria on the stiffer glioma simulating substrate than on the softer brain simulating substrate, in both 2-D and 3-D constructs $(p<0.05)$. But at any given substrate stiffness, U118 did not show a significant difference in mitochondrial PPIX concentration between the 2$\mathrm{D}$ and the 3-D constructs. On the other hand, PPIX production in U373 seemed to be less sensitive to the gel substrate conditions studied here. No significant change in U373 mitochondrial
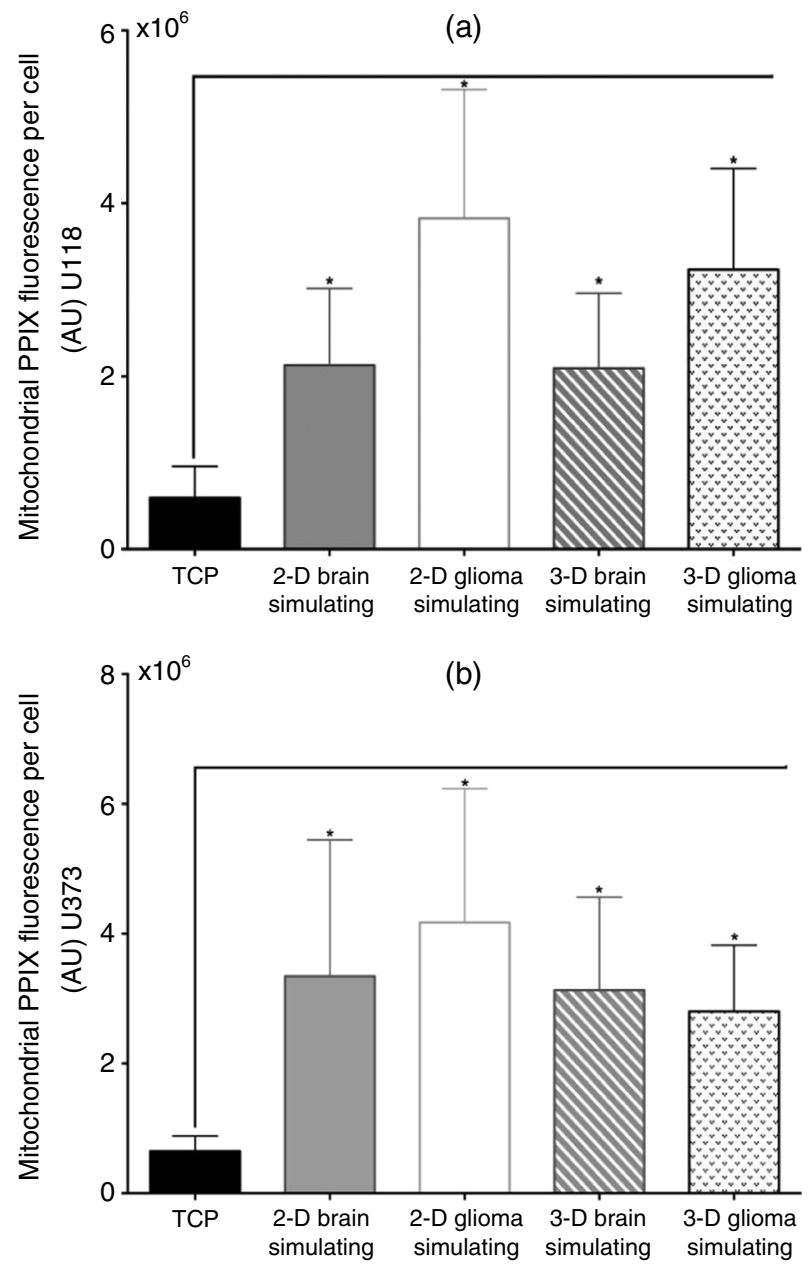

Fig. 3 Average protoporphyrin IX fluorescence counts in the mitochondria per cell in glioma cells cultured under five substrate conditions: TCP, 2-D simulating gel, 2-D glioma simulating gel, threedimensional (3-D) brain simulating gel, and 3-D glioma simulating gel. Statistical analysis was performed on each of the four gel conditions against TCP $\left({ }^{*} p<0.05\right)$ : (a) U118 and (b) U373.

PPIX concentration was observed between any two gel-made conditions.

Interestingly, the pattern of PPIX production in U118 and U373 on different substrates appeared to correspond to the two cell lines' cell density increase rates on the substrates. Just like the results of the apparent growth rate experiment, the stiffness increase from brain to glioma simulating gel seemed to have a consistent effect on the PPIX synthesis in U118, which produced more PPIX on the modestly stiffer glioma simulating gel $(\sim 12 \mathrm{kPa})$ than on the softer brain simulating gel $(\sim 1 \mathrm{kPa})$. This holds true for both the 2-D and 3-D environments. Again, U118 appeared to be sensitive to the change in substrate stiffness within the range of 1 to $12 \mathrm{kPa}$. While U118 in a biologically relevant mechanical environment exhibits faster apparent proliferation, an indication of increased malignancy, it is encouraging that it also demonstrated enhanced PPIX production, as seen in the increased PPIX fluorescence count on the gel substrates compared to the rigid TCP. This consequently means a stronger PPIX mediated PDT efficacy could be achieved in the actual bulk tumor in vivo than the PDT efficacy observed on a rigid TCP surface in vitro. The situation is different for cells invading into the brain adjacent to tumor as they will reside in tissues with 
a lower Young's modulus, which, based on the observation made here, would possibly lead to limited PPIX accumulation, and consequently a weaker signal for fluorescence guided resection and a lower PDT dose delivered to these cells. On the other hand, the stiffness difference between the brain and glioma simulating gels did not appear to lead to any statistically significant change in PPIX synthesis in U373. Thus, the Young's moduli of the tumor and the normal neuronal tissue represent a previously unknown potential influence on PPIX synthesis, which should be taken into consideration when developing new techniques of quantitative fluorescence guided resection.

Unfortunately, a literature search did not offer much insight on the potential mechanisms through which substrate stiffness could affect PPIX synthesis. However, some publications exploring the impact of substrate stiffness on various other cellular activities may provide some clues on the possible mechanisms of how matrix stiffness can influence PPIX synthesis. For example, Ulrich et al. observed that from TCP to gel substrate whose Young's modulus went as low as $0.1 \mathrm{kPa}$, glioma cells showed reduced proliferation and migration. The cells also adapted a more energy-efficient spherical shape on softer gels over a more energy-consuming flattened morphology on a rigid surface. ${ }^{27}$ One can logically deduct that the energy saved from slower proliferation and migration could be spent elsewhere, including an increase in mitochondrial activity, which could in turn lead to an increase in PPIX synthesis.

For both cell lines, cell-to-cell variations in the mitochondrial PPIX concentration were observed, probably due to differences in the ability to synthesize PPIX at different stages of the cell cycle. $^{39}$ Mitochondrial PPIX concentration had a larger fluctuation on gel substrates than on TCP, as indicated by the larger standard deviation. A probable explanation is that the change in mechanical environment altered the cells' metabolic activities, leading to an increased cell-to-cell variation in PPIX production, accumulation, and distribution. Given the tendency of these two glioma cells to grow into spheroids on soft substrates, confocal microscopy is probably more suitable than wide-field microscopy to examine PPIX concentration and even more so intracellular distribution of PPIX. As the Young's modulus of glioma tumors can range from a few $\mathrm{kPa}$ to $>40 \mathrm{kPa},{ }^{40}$ one may need to expand the range of Young's moduli of the substrate material in future studies. It would also be worthwhile to attempt to construct an environment that is not only biomechanically but also biochemically relevant to the actual ECM in the brain, since neuronal ECM has numerous distinctive characteristics such as extra abundance of hyaluronic acid and apparent lack of collagen compared to other tissues in the human body. The uniqueness in brain ECM composition is believed to partially contribute to some of the unusual behaviors of glioma, such as rare invasion into the vasculatures. ${ }^{41}$ While it is certainly important to study glioma cells on tissue modeling substrates, in vitro examination of normal neuronal cells in a biologically relevant environment should not be overlooked. How far a change in PPIX cellular concentration impacts on the PDT response is currently under investigation.

\section{Conclusions}

This work presents a starting point of probing the effects of biomechanical factors in addition to the already known chemical and biochemical microenvironmental factors that can impact on the efficacy of both FGR and PDT in the management of glioma. The results, albeit preliminary, highlight the importance of creating biologically relevant mechanical environments in vitro in order to accurately evaluate cell behavior, response to PDT, and fluorescence contrast for FGR.

In agreement with previously published studies, the overall trend is that glioma cells proliferate faster on stiffer substrates. However, the degree of this effect on cell proliferation appeared to be more complicated than a straightforward linear relationship. U118 appeared to proliferate faster as the substrate became stiffer, but not significantly given the data collected in this investigation. In U373, the substrate stiffness did not seem to significantly promote cell proliferation until reaching a threshold Young's modulus, which can be exploited by the cytoskeleton and the filapodia. More so, this threshold seems to apply to the effect of substrate stiffness on average PPIX synthesis per cell, probably due to a range of cellular mechanisms activated at the threshold substrate stiffness, which may be involved in both proliferation and PPIX synthesis. However, this link between proliferation and PPIX synthesis/retention did not extend to the extreme stiffness of TCP, on which cells divided the fastest but produced the least amount of PPIX.

When using ALA induced PPIX for FGR, in addition to physical and biological fluorescence intensity modifiers, including the variation in tissue optical properties and glioma cell density in the bulk tumor and the adjacent brain tissue, it may be important to also look at the Young's modulus of the target tissue, which may contribute to a more accurate estimation of the PPIX synthesis rate that will impact on the fluorescent signal. If this is the case, biomechanical properties would be another factor to consider when developing quantitative fluorescence guided resection.

\section{Acknowledgments}

This study was funded in part by the Canadian Institute of Health Research and the Ontario Ministry of Health and Long Term Care.

\section{References}

1. H. Kostron, "Photodynamic diagnosis and therapy and the brain," in Photodynamic Therapy-Methods and Protocols, C. J. Gomer, Ed., pp. 261-280, Humana Press, New York (2010).

2. C. J. Fisher and L. Lilge, "Photodynamic therapy in the treatment of intracranial gliomas: a review of current practice and considerations for future clinical directions," J. Innov. Opt. Health Sci. 8(1), 1530005 (2015)

3. S. S. Stylli and A. H. Kaye, "Photodynamic therapy of cerebral glioma-a review. Part II-clinical studies," J. Clin. Neurosci. 13, 709-717 (2006).

4. W. Stummer et al., "Long-sustaining response in a patient with nonresectable, distant recurrence of glioblastoma multiforme treated by interstitial photodynamic therapy using 5-ALA: case report," $J$. Neuro-Oncology 87(1), 103-109 (2008).

5. T. J. Beck et al., "Interstitial photodynamic therapy of nonresectable malignant glioma recurrences using 5-aminolevulinic acid induced protoporphyrin IX," Lasers Surg. Med. 39, 386-393 (2007).

6. Y. Muragaki et al., "Phase II clinical study on intraoperative photodynamic therapy with talaporfin sodium and semiconductor laser in patients with malignant brain tumors," J. Neurosurg. 119, 845-852 (2013).

7. J. C. Kennedy, R. H. Pottier, and D. C. Pross, "Photodynamic therapy with endogenous protoporphyrin IX: basic principles and present clinical experience," J. Photochem. Photobiol. 6, 143-148 (1990).

8. B. C. Wilson, M. S. Patterson, and L. Lilge, "Implicit and explicit dosimetry in photodynamic therapy-a new paradigm," Lasers Med. Sci. 12(3), 182-199 (1997). 
9. L. Lilge and B. C. Wilson, "Photodynamic therapy of intracranial tissues: a preclinical comparative study of four different photosensitizers," J. Clin. Laser Med. Surg. 16(2), 81-91 (1998).

10. A. Kim et al., "Quantification of in vivo fluorescence decoupled from the effects of tissue optical properties using fiber-optic spectroscopy measurements," J. Biomed. Opt. 15(6), 067006 (2010).

11. U. Sunar et al., "Quantification of PpIX concentration in basal cell carcinoma and squamous cell carcinoma models using spatial frequency domain imaging," Biomed. Opt. Express 4(4), 531-537 (2013).

12. W. Stummer et al., "5-aminolevulinic acid-derived tumor fluorescence: the diagnostic accuracy of visible fluorescence qualities as corroborated by spectrometry and histology and postoperative imaging," Neurosurgery 74, 310-320 (2014).

13. L. Wyld, M. W. R. Reed, and N. J. Brown, "The influence of hypoxia and $\mathrm{pH}$ on aminolevulinic acid-induced photodynamic therapy in bladder cancer cells in vitro," Br. J. Cancer 77(10), 1621-1627 (1998).

14. P. Uehlinger et al., "5-aminolevulinic acid and its derivatives: physical chemical properties and protoporphyrin IX formation in cultured cells," J. Photochem. Photobiol. 54, 72-80 (2000).

15. C. J. Fisher et al., "Modulation of PPIX synthesis and accumulation in various normal and glioma cell lines by modification of the cellular signaling and temperature," Lasers Surg. Med. 45, 460-468 (2013).

16. A. Johansson et al., "Protoporphyrin IX fluorescence and photobleaching during interstitial photodynamic therapy of malignant gliomas for early treatment prognosis," Lasers Surg. Med. 45, 225-234 (2013).

17. P. Valdes et al., "5-aminolevulinic acid-induced protoporphyrin IX concentration correlates with histopathologic markers of malignancyin human gliomas: the need for quantitative fluorescence-guided resection to identify regions of increasing malignancy," Neuro-Oncology 13(8), 846-856 (2011)

18. P. A. Valdés et al., "Quantitative, spectrally-resolved intraoperative fluorescence imaging," Sci. Rep. 2, 798 (2012).

19. A. Bogaards et al., "Increased brain tumor resection using fluorescence image guidance in a preclinical model," Lasers Surg. Med. 35(3), 181190 (2004).

20. C. Frantz, K. M. Stewart, and V. M. Weaver, "The extracellular matrix at a glance," J. Cell Sci. 123, 4195-4200 (2010).

21. T. Amo et al., "Mechanism of cell death by 5 -aminolevulinic acid-based photodynamic action and its enhancement by ferrochelatase inhibitors in human histiocytic lymphoma cell line U937," Cell Biochem. Funct. 27(8), 503-515 (2009).

22. H. Kobuchi et al., "Mitochondrial localization of $\mathrm{ABC}$ transporter $\mathrm{ABCG} 2$ and its function in 5-aminolevulinic acid-mediated protoporphyrin IX accumulation," PLoS One 7(11), e50082 (2012).

23. M. Yamamoto et al., "Improvement of the efficacy of 5-aminolevulinic acid-mediated photodynamic treatment in human oral squamous cell carcinoma HSC-4," Acta Med. Okayama 67(3), 153-164 (2013).

24. D. E. Discher, P. Janmey, and Y.-1. Wang, "Tissue cells feel and respond to the stiffness of their substrate," Science 310(5751), 1139-1143 (2005).

25. A. J. Engler et al., "Matrix elasticity directs stem cell lineage specification," Cell 126, 677-689 (2006).

26. T. R. Cox and J. T. Erler, "Remodeling and homeostasis of the extracellular matrix: implications for fibrotic diseases and cancer," Dis. Model. Mech. 4(2), 165-178 (2011).

27. T. A. Ulrich, E. M. d. J. Pardo, and S. Kumar, "The mechanical rigidity of the extracellular matrix regulates the structure, motility, and proliferation of glioma cells," Cancer Res. 69(10), 4167-4174 (2009).

28. M. C. Murphy et al., "Decreased brain stiffness in Alzheimer's disease determined by magnetic resonance elastography," J. Magn. Reson. Imaging 34(3), 494-498 (2011).
29. R. S. Fischer et al., "Stiffness-controlled three-dimensional extracellular matrices for high-resolution imaging of cell behavior," Nat. Protocol 7(11), 2056-2066 (2012).

30. H. Liu, Y. Sun, and C. A. Simmons, "Determination of local and global elastic moduli of valve interstitial cells cultured on soft substrates," $J$. Biomech. 46(11), 1967-1971 (2013).

31. P. K. Kennady et al., "Variation of mitochondrial size during the cell cycle: a multiparameter flow cytometric and microscopic study," Cytometry A 62(2), 97-108 (2004).

32. N. Otsu, "A threshol selection method from gray-level histograms," IEEE Trans. Syst. Man Cybern. 9(1), 62-66 (1979).

33. C. Wang, X. Tong, and F. Yang, "Bioengineered 3D brain tumor model to elucidate the effects of matrix stiffness on glioblastoma cell behavior using PEG-based hydrogels," Mol. Pharm. 11(7), 2115-2125 (2014).

34. V. Umesh et al., "Microenvironmental stiffness enhances glioma cell proliferation by stimulating epidermal growth factor receptor signaling," PLoS One 9(7), e101771 (2014).

35. U. Andersson et al., "Epidermal growth factor receptor family (EGFR, ErbB2-4) in gliomas and meningiomas," Acta Neuropathol. 108, 135-142 (2004).

36. A. Hadj Henni, C. Schmitt, and G. Cloutier, "Shear wave induced resonance elastography of soft heterogeneous media," J. Biomech. 43, 1488-1493 (2010).

37. R. Sinkus et al., "Viscoelastic shear properties of in vivo breast lesions measured by MR elastography," Magn. Reson. Imaging 23, 159-165 (2005).

38. C. Sun, B. Standish, and V. X. D. Yang, "Optical coherence elastography: current status and future applications," J. Biomed. Opt. 16(4), 043001 (2011).

39. L. Wyld et al., "Cell cycle phase influences tumour cell sensitivity to aminolevulinic acid-induced photodynamic therapy in vitro," $\mathrm{Br}$. $J$. Cancer 78(1), 50-55 (1998).

40. A. J. Palocaren and C. S. Drapaca, "Biomechanical modeling of tumor growth: its relevance to glioma research," Int. J. Numer. Anal. Model. B 3(1), 94-108 (2012).

41. B. Ananthanarayanan, Y. Kim, and S. Kumar, "Elucidating the mechanobiology of malignant brain tumors using a brain matrix-mimetic hyaluronic acid hydrogel platform," Biomaterials 32(11), 7913-7923 (2011).

Carl Fisher received his BSc degree in biochemistry (honours) at McGill University, Montreal, Quebec, Canada, in 2009. Since 2009, he has entered into graduate studies in the Department of Medical Biophysics at the University of Toronto, working towards his PhD. His research interests include examining how modifications to cellular sensitivity can improve upon current PDT treatments of glioma, and additionally using MRI techniques to determine if patient tumours are suitable for PDT treatment.

Lothar Lilge completed his training in Germany (Goethe University and Wilhelms University), the USA (Massachusetts General Hospital) and Canada (McMaster University). Since 2009, he is a senior staff scientist at the Princess Margaret Cancer Centre and professor at the University of Toronto. His research interest centers on photodynamic therapy, microfluidics and cancer risk assessment.

Biographies for the other authors are not available. 